\title{
Die Niere als Vollstrecker der Therapie bei Typ-2-Diabetes
}

\section{Liebe Leserinnen und Leser,}

die komplexe Pathophysiologie des Diabetes mellitus Typ 2 bedingt, dass solche Therapieregime die größte Aussicht auf Erfolg haben, die unterschiedliche Wirkansätze additiv miteinander kombinieren. Obwohl derzeit bereits viele orale Antidiabetika und Insulinpräparate für eine effektive Differenzialtherapie zur Verfügung stehen, sind diese jedoch im Wesentlichen auf die symptomatische Senkung der Blutglukosespiegel ausgerichtet. Hiermit gelingt es nicht, zuverlässig bei jedem Patienten diabetische Folgeerkrankungen zu verhindern. Darüber hinaus werden heute wesentlich höhere Anforderungen an antihyperglykämische Medikamente gestellt. Über die Fähigkeit zur reinen Blutzuckersenkung hinaus wird von den Zulassungsbehörden vermehrt der Nachweis der kardiovaskulären Sicherheit, bzw. sogar die Reduktion harter patientenrelevanter Endpunkte bei Typ-2-Diabetikern mit hohem kardiovaskulären Risiko gefordert. Darüber hinaus wird deutlich, dass der Erhalt der residuellen Betazellfunktion und damit die Langzeit-Glukosekontrolle und Progressionsverzögerung der Typ-2Diabetes-Erkrankung als zukünftig wichtiges Kriterium von Medikamenten und deren Kombinationen gewertet werden wird.

\section{Die „therapeutische Glukosurie“ hat viele angenehme Begleiteffekte...}

Trotz der hohen Anforderungen an die Zulassung neuer oraler Antidiabetika ist die „Pipeline“ für Antidiabetika mit innovativen Wirkansätzen gut gefüllt.Wie im CME-Beitrag von Martin in diesem Heft ausgeführt, werden hier auch medikamentöse Ansätze mit „neuen“ Zielorganen, in diesem speziellen Fall der Niere, verfolgt. Hierbei müssen wir als Diabetologen lernen, dass die Niere nicht nur als geschädigtes Organ im Rahmen der Komplikationen des Diabetes mellitus gesehen werden darf. Wir müssen uns die seit langem bekannte, aber etwas in Vergessenheit geratene aktive Rolle der Niere bei der Regulation der Glukosehomöostase wieder neu vor Augen führen. Die medikamentöse Beeinflussung dieser regulatorischen Funktion im Sinne der Induktion einer "therapeutischen Glukosurie“ durch Hemmung des Natrium-Glukose-Kotransporters Typ 2 (SGLT-2) auf der Nierentubuluszelle liegt dem Wirkmechanismus neuer oraler Anti- diabetika zugrunde.Diese sogenannten „Gliflozine“ (Dapagliflozin, Remogliflozin, Canagliflozin, Sergliflozin, Empagliflozin) befinden sich derzeit in weit fortgeschrittener klinischer Entwicklung und werden die nächste Substanzklasse oraler Antidiabetika mit neuem Wirkprinzip darstellen. Diese Medikamente sind neben dem Effekt auf die Glykämie zusätzlich interessant bei Typ-2-Diabetes, weil sie einen gewichtsreduzierenden Effekt haben (Glukosurie bedingt Energieverlust über die Niere), nur ein geringes Hypoglykämierisiko bergen und den Blutdruck senken (durch Natriumverlust über die Niere). Zudem besteht die potenzielle Einsatzmöglichkeit als erste orale Antidiabetika zur Behandlung bei Typ-1-Diabetes.

Aktuelle Studiendaten bei Typ-2-Diabetes zeigen eine $\mathrm{HbA}_{1 \mathrm{c}}$-Senkung von etwa $0,6-1 \%$ in der Monotherapie (siehe Beitrag von Martin in diesem Heft). Da die Wirkung komplett vom Insulin unabhängig erfolgt, könnten die Gliflozine - zumindest theoretisch - sinnvoll in der Kombinationstherapie in jedem Stadium des Typ-2-Diabetes eingesetzt werden. Allerdings ist aufgrund des Wirkmechanismus bei einer stark eingeschränkten glomerulären Filtrationsrate bei Niereninsuffizienz mit einem deutlichen Wirkverlust zu rechnen.

\section{...und einige weniger angenehme Begleiteffekte}

Bedenken gegen die pharmakologische Erhöhung der Harnglukose durch diese Substanzen gründen sich vor allem auf die Befürchtung, dass hierdurch vermehrt Infekte der ableitenden Harnwege und der Genitalien induziert werden könnten, für die Diabetiker sowieso prädestiniert sind. In den derzeit laufenden Zulassungsstudien wird tatsächlich konsistent eine erhöhte Rate an Genitalinfektionen verzeichnet, besonders bei Frauen. Diese Nebenwirkung könnte eine Kontraindikation für den Langzeiteinsatz bei prädestinierten Patient(inn)en sein.

Dennoch sind die SGLT-2-Inhibitoren ein innovativer Ansatz zur additiven Behandlung von Menschen mit Typ-2-Diabetes in jedem Stadium der Erkrankung, falls aktuelle Studien keine weiteren Toxizitäten und Langzeit-Nebenwirkungen zutage fördern.

Ihr Jochen Seufert

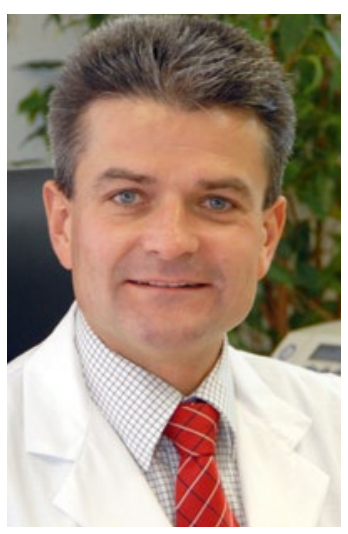

Prof. Dr. med. Jochen Seufert, FRCPE Schwerpunktleiter Endokrinologie und Diabetologie Abteilung Innere Medizin II Universitätsklinikum Freiburg 